\title{
Pattern formation in growing sandpiles
}

\author{
Deepak Dhar ${ }^{*}$ Tridib Sadhu周 and Samarth Chandra周 \\ Department of Theoretical Physics, Tata Institute of Fundamental Research, 1 Homi Bhaba Road, Mumbai 400005 India
}

(Dated: October 23, 2019)

\begin{abstract}
Adding grains at a single site on a flat substrate in the Abelian sandpile models produce beautiful complex patterns. We study in detail the pattern produced by adding grains on a two-dimensional square lattice with directed edges (each site has two arrows directed inward and two outward), starting with a periodic background with half the sites occupied. The size of the pattern formed scales with the number of grains added $N$ as $\sqrt{N}$. We give exact characterization of the asymptotic pattern, in terms of the position and shape of different features of the pattern.
\end{abstract}

Many complicated and intricate patterns found in nature can be modelled by deterministic dynamics 1 . In Turing patterns [2] the final outcome is random due to the randomness in initial conditions. In the game of life [3], one can get a very wide variety of patterns from simple deterministic cellular automaton evolution rules, depending on the initial condition.

While the real sand, poured at one point on a flat substrate produces a rather simple pyramidal shape, much more complex patterns are produced in the theoretical models of sandpiles, like the Abelian sandpile model (ASM) 4. Earlier studies have usually concentrated on determining the asymptotic shape of the growing cluster [5, 6. Other special configurations in the model, like the identity [7, or the stable state produced from special unstable states also show complex internal self-similar structures [8]. The limiting shape has been determined in the related rotor-router model, and the model of divisible sandpiles with multiple sites of addition [9].

In this paper, we study the asymptotic pattern produced by adding $N$ grains of sand at a single site on a two dimensional Abelian sandpile model starting from a periodic background, and allowing the system to relax. It is easy to see that the diameter of the pattern grows as $\sqrt{N}$. Interestingly, for large $N$, the pattern shows a proportionate growth, with different parts of the pattern all growing as $\sqrt{N}$. This is thus different from earlierstudied models of growth such as diffusion limited aggregation, Eden model etc. [10, where the growth occurs mainly at the surface.

The standard square lattice produces a rather complicated pattern (Fig $1 a$ ), and it has not been possible to characterize it so far. We consider two variations, assigning orientations to the edges of the lattice, as shown in Fig $2 a$ and $2 p$. The initial state was chosen to be a periodic checkerboard arrangement of sites with heights 0 and 1 . The asymptotic pattern produced in the two cases turns out to be the same, and is shown in Fig 1p. Taking some qualitative features of the observed pattern ( e.g. only two types of patches are present, and they are all 3- or 4- sided polygons) as input, we show how one can get a complete and quantitative characterization of the pattern. We show that the pattern has exact 8-fold rotational symmetry, and determine the exact coordinates
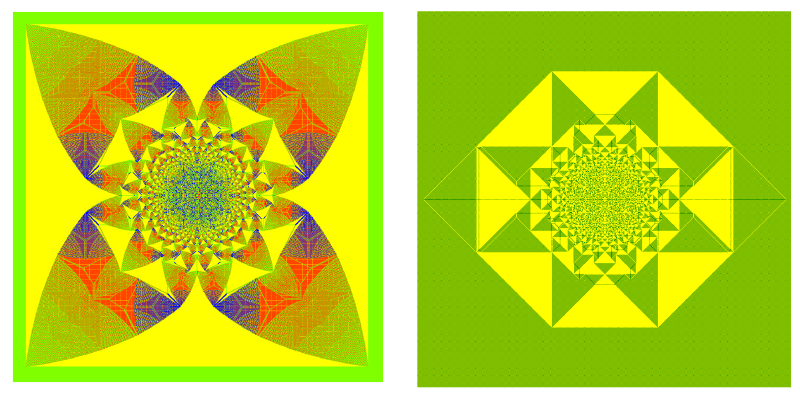

FIG. 1: Stable configurations for the Abelian sandpile model obtained by adding particles at one site. (a) Undirected square lattice, initial configurations with all heights 2 , and $2 \times 10^{5}$ particles added, color code: red $=0$, blue $=1$, green $=2$, yellow $=3$. (b) The F-lattice of Fig $2 a$ with initial checkerboard configuration, with $2 \times 10^{5}$ particles added, color code: green $=0$, yellow $=1$. The apparent green regions in the picture represent the patches with checkerboard configuration.
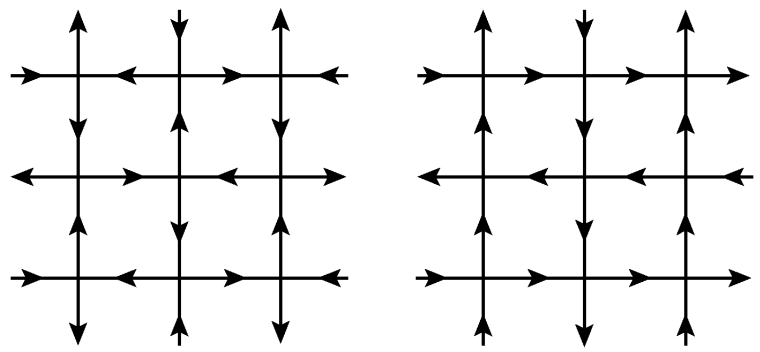

FIG. 2: The directed square lattices studied in this paper $(a)$ the F-lattice $(b)$ the Manhattan lattice.

of all the boundaries in the asymptotic pattern. We discuss some other cases, where exactly the same pattern is obtained.

In the two lattices we studied (Fig 2), each bond of the lattice is directed with two in-arrows, and two outarrows at each vertex. The ASM on these is defined by the toppling rule: A site $(x, y)$ is unstable if the number of grains at the site $z_{x, y} \geq 2$, and then transfers one grain each in the direction of its outward arrows. We start with an initial configuration in which $z_{x, y}=1$, for sites with $(x+y)=$ even, and 0 otherwise. 
We used a lattice large enough so that no avalanches started from the origin reach the boundary. Using the Abelian property, we add all $N$ particles in the beginning, and relax the configuration to get the final pattern. The result of adding $N=2 \times 10^{5}$ particles on the F-lattice is shown in Fig $1 p$. The pattern formed on the Manhattan lattice is indistinguishable at large scales. The pattern is identical to Fig.1p, except that the thin lines of 1's forming two triangles outside the octagon are rotated by $45^{\circ}$ in the Manhattan case. Since the lattices are quite different, this is quite intriguing.

We start by setting up some general theoretical framework, which is independent of the details of the particular lattices studied. Formally, we can characterize the asymptotic pattern in terms of the rescaled coordinates, $\xi=x / \sqrt{N}, \eta=y / \sqrt{N}$ and the density function $\rho(\xi, \eta)$ which gives the local density of grains in the pattern in a small rectangle of size $\Delta \xi, \Delta \eta$ about the point $(\xi, \eta)$, with $N^{-1 / 2} \ll \Delta \xi, \Delta \eta \ll 1$.

Equivalently, we can describe the asymptotic pattern in term of the rescaled toppling function $\phi(\xi, \eta)$. Let $T_{N}(x, y)$ be the numbers of toppling at site $(x, y)$ when $N$ particles are added at the origin, and the configuration is relaxed. We define

$$
\phi(\xi, \eta)=\lim _{N \rightarrow \infty} \frac{1}{2 N} T_{N}(\lfloor\sqrt{N} \xi\rfloor,\lfloor\sqrt{N} \eta\rfloor)
$$

where floor function $\lfloor x\rfloor$ is the largest integer less than $x$. From the conservation of sand grains, it is easily seen that $\phi(\xi, \eta)$ is related to the density function $\rho(\xi, \eta)$ by

$$
\left(\frac{\delta^{2}}{\delta \xi^{2}}+\frac{\delta^{2}}{\delta \eta^{2}}\right) \phi(\xi, \eta)=\Delta \rho(\xi, \eta)-\delta(\xi) \delta(\eta)
$$

where excess density $\Delta \rho(\xi, \eta)$ is the difference between $\rho(\xi, \eta)$ and the initial density $\rho_{0}(\xi, \eta)$.

It was already noted [11] that for $N$ large $\rho(\xi, \eta)$ tends to a nontrivial limit, and the asymptotic pattern is made of distinct regions, called 'patches'. Typically inside a patch the heights are periodic in space, and there are few defect-lines, which move with $N$, but do not change the macroscopic density $\rho(\xi, \eta)$. Then, the coarse grained function $\rho(\xi, \eta)$ takes constant rational value in each patch. Also in each patch of constant $\Delta \rho(\xi, \eta), \phi(\xi, \eta)$ is a quadratic function, and was first noted in [11. We indicate the proof here. For all patches the function $\phi(\xi, \eta)$ is Taylor expandable around any point inside the patch. Consider any term of order $\geq 3$ in the expansion, for example the term $\sim(\Delta \xi)^{3}$. This can only arise due to a term $\sim(\Delta x)^{3} / \sqrt{N}$ in $T(x, y)$. Then the integer function $T(x, y)$ will change discontinuously at intervals of $\Delta x \sim \mathcal{O}\left(N^{1 / 6}\right)$ leading to infinitely many defect-lines in the asymptotic pattern. However there are no such feature in Fig 1 7 or Fig 1 1 . Therefore inside a patch of constant $\Delta \rho(\xi, \eta), \phi(\xi, \eta)$ can at most be quadratic in $\xi$ and $\eta$, and in each periodic patch, the toppling function $T(x, y)$ is sum of two terms: a part that is a simple quadratic function of $x$ and $y$, and a periodic part.
The periodic part averages to zero, and does not contribute to the coarse-grained function $\phi(\xi, \eta)$. In some patterns, there are regions of finite fractional area which show aperiodic height patterns. In these regions $\phi(\xi, \eta)$ is not quadratic and are harder to characterize.

Now consider two neighboring periodic patches $\mathbf{P}$ and $\mathbf{P}^{\prime}$ with mean densities $\rho$ and $\rho^{\prime}$ respectively. Let the quadratic toppling function be $Q(\xi, \eta)$ and $Q^{\prime}(\xi, \eta)$ in these patches. Then the boundary between the patches is given by the equation $Q(\xi, \eta)=Q^{\prime}(\xi, \eta)$. As the derivatives of $\phi$ are also continuous across the boundary, the boundary between two periodic patches must be a straight line, and

$$
Q^{\prime}(\xi, \eta)=Q(\xi, \eta)+\frac{1}{2}\left(\rho^{\prime}-\rho\right) l_{\perp}^{2}
$$

where $l_{\perp}$ is the perpendicular distance of $(\xi, \eta)$ from the boundary. We can start with a periodic patch $P$, and go to another patch $P^{\prime}$ by more than one path. Since the final quadratic function at $P^{\prime}$ should be the same whichever path we take, this imposes consistency conditions which restricts the allowed values of slopes of boundaries. Consider a point $z_{0}$ where $n$ periodic patches meet, with $n>2(\operatorname{Fig} 3 a)$. If the $j$ th boundary at this point makes an angle $\theta_{j}$ with the $x$-axis, and the density of the patch in the wedge $\theta_{j} \leq \theta \leq \theta_{j+1}$ is $\rho_{j+1}$ (Fig] $3 a$ ) then using Eq3 repeatedly for all $n$ patches around $z_{0}$ we get that the following equation must be true for all $\theta$ :

$$
\sum_{j=1}^{n}\left(\rho_{j+1}-\rho_{j}\right) \sin ^{2}\left(\theta-\theta_{j}\right)=0
$$

with $\rho_{n+1}=\rho_{n}$. This is equivalent to the condition:

$$
\sum_{j=1}^{n}\left(\rho_{j+1}-\rho_{j}\right) e^{2 i \theta_{j}}=0
$$

For $n=3$, with $\rho_{1} \neq \rho_{2} \neq \rho_{3}$, this equation has only trivial solutions with $\theta_{j}$ equal to 0 or $\pi$ for all $j$. Hence, only $n \geq 4$ are allowed.

We now discuss how the exact function $\rho(\xi, \eta)$ can be determined for our problem. We note that in Fig 13 , there are no aperiodic patches, only two types of periodic patches, where $\rho(\xi, \eta)$ only take values 1 or $1 / 2$. Also, the slopes of the boundaries between patches only take values $0, \pm 1, \infty$. The patches are typically dart shaped quadrilaterals, and some triangles (which may be considered as degenerate quadrilaterals with one side of length zero). These simplifications, not present in Fig 1 1 a, make possible a full characterization of the pattern in Fig $1 p$.

Given that there are only these two types of patches, we only need to look for possible patterns where $\Delta \rho$ takes piecewise constant values $1 / 2$ or 0 . From Eq.(2), we see that we can think of $\phi(\xi, \eta)$ as the potential produced by a point charge at the origin, and a charge cloud with 

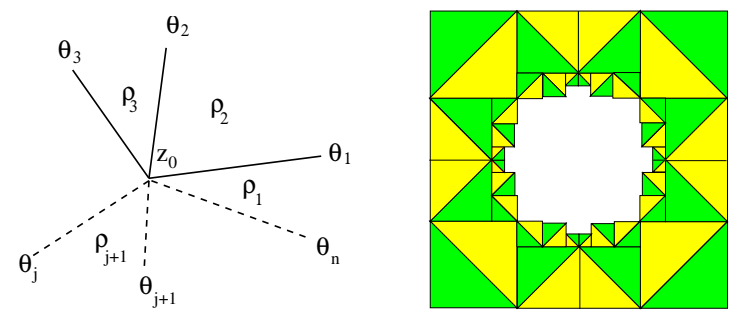

FIG. 3: (a) $n$ different periodic patches of density $\rho_{1}, \ldots, \rho_{n}$ meeting at point $z_{0}$. (b) The pattern in Fig 1 p is obtainable by putting together square tiles of different sizes. Each of the tiles is divided into two halves of different density.

areal density $-\Delta \rho(\xi, \eta)$, with total charge zero. The basic principle which selects the actual stable pattern out of many is a version of the principle of minimum dissipation: It is a stable state reached by minimum number of toppling. (This follows immediately from the toppling rules, where no toppling occurs unless forced).

The requirement that $\phi(\xi, \eta)$ be exactly zero, in the region outside the pattern, implies that all the multipole moments of the charge distribution $\Delta \rho(\xi, \eta)$ are exactly zero. We show below that the conditions that $\Delta \rho$ takes only two values, the potential function is exactly quadratic within a periodic patch, and the slopes of the boundaries are only $0, \pm 1, \infty$, fix the allowed pattern uniquely.

We start by determining the exact asymptotic size of the pattern. We note from Fig $1 p$ that the boundary of the pattern is an octagon ( we shall prove later that this is a regular octagon ). In fact there are four lines of 1's outside the octagon. But these has zero areal density in the limit $N \rightarrow \infty$, and do not contribute to $\rho(\xi, \eta)$. We will ignore these in the following discussion.

Let $B$ be the minimum boundary square containing all $(\xi, \eta)$ that have a non-zero charge density $\rho(\xi, \eta)$. We observe that $B$ can be considered as a union of disjoint smaller squares, each of which is divided by diagonal into two parts where $\Delta \rho(\xi, \eta)$ takes values $1 / 2$ and 0 [Fig $3 p]$. This is seen to be true for the outer layer patches. Towards the center, the squares are not so well resolved. Assuming that this construction remains true all the way to the center, in the limit of large $N$, the mean density of the negative charge in the bounding square $=1 / 4$. Given that the total amount of negative charge is -1 , the area of the bounding square should be 4 . Hence we conclude that the equation of the boundary of the minimum bounding square are

$$
|\xi|=1, \quad|\eta|=1
$$

Let $N_{b}$ be the minimum number of particles that have to be added so that at least one site at $y=b$ topples. We find that for $b=10,50,100$, and $300, \sqrt{N_{b}}=10.770$, $49.436,98.894$ and 297.798. Clearly the boundary distance $b$ tends to $\sqrt{N}$ for large $N$.

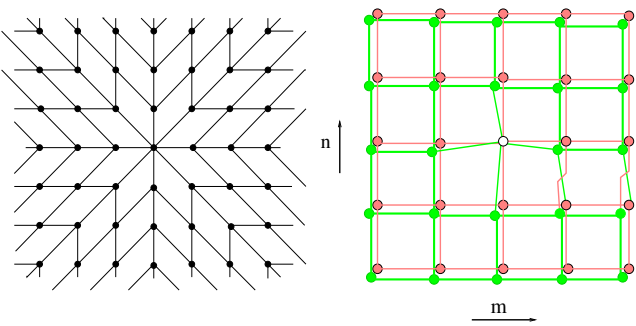

FIG. 4: Two representations of the adjacency graph of the pattern. Here the vertices are the patches, and the edges connect the adjacent patches. (a) Representation as a planar graph (b) as a graph of wedge of angle $4 \pi$ formed by glueing together the eight quadrant graphs at the origin.

We now describe the topological structure of the pattern. This is characterized by its adjacency graph $[\mathrm{Fig}[4 \mathrm{a}]$, where each vertex denotes a patch, and a bond between the vertices is drawn if the vertices share a common boundary. It is convenient to think of the triangular patches in the pattern as degenerate quadrilaterals, with one side of length zero. Then we see that the adjacency graph is planar with each vertex of degree four, except a single vertex of coordination number eight corresponding to the exterior of the pattern. The graph has the structure of a square lattice wedge, with wedge angle $4 \pi$. The square lattice structure of the adjacency graph is seen most directly by applying a $z^{\prime}=1 / z^{2}$ transformation to the picture (used earlier in 11), where $z=\xi+i \eta$, and view it in the complex $z^{\prime}$-plane. Thus, one can equivalently represent the graph as a square grid on a Riemann surface of two sheets (fig, $4 \beta$ ).

We now use the qualitative information obtained from the adjacency matrix of the observed pattern, to obtain quantitative prediction of the exact coordinates of all the patches. Consider an arbitrary patch $\mathbf{P}$, having an excess density $1 / 2$. The potential function in the patch is a quadratic function of $(\xi, \eta)$ and we parametrize it as

$$
\begin{aligned}
\phi_{\mathbf{P}}(\xi, \eta)= & \frac{1}{8}\left(m_{\mathbf{P}}+1\right) \xi^{2}+\frac{1}{4} n_{\mathbf{P}} \xi \eta+\frac{1}{8}\left(1-m_{\mathbf{P}}\right) \eta_{p}^{2} \\
& +d_{\mathbf{P}} \xi+e_{\mathbf{P}} \eta+f_{\mathbf{P}}
\end{aligned}
$$

The potential function in a patch $\mathbf{P}$ having zero excess density will be parametrized as

$$
\phi_{\mathbf{P}}(\xi, \eta)=\frac{1}{8} m_{\mathbf{P}}\left(\xi^{2}-\eta^{2}\right)+\frac{1}{4} n_{\mathbf{P}} \xi \eta+d_{\mathbf{P}} \xi+e_{\mathbf{P}} \eta+f_{\mathbf{P}}
$$

Now consider two neighboring patches $\mathbf{P}$ and $\mathbf{P}^{\prime}$ with excess densities $1 / 2$ and 0 respectively. Then using the matching condition Eq.(3), it is easy to show that if the boundary between them is a horizontal line $\eta=\eta_{\mathbf{P}}$, we must have

$$
\begin{aligned}
m_{\mathbf{P}^{\prime}} & =m_{\mathbf{P}}+1, \quad \mathrm{n}_{\mathbf{P}^{\prime}}=\mathrm{n}_{\mathbf{P}}, \quad \mathrm{d}_{\mathbf{P}^{\prime}}=\mathrm{d}_{\mathbf{P}} \\
e_{\mathbf{P}^{\prime}} & =e_{\mathbf{P}}+\eta_{\mathbf{P}} / 2, \quad \mathrm{f}_{\mathbf{P}^{\prime}}=\mathrm{f}_{\mathbf{P}}-\eta_{\mathbf{P}}^{2} / 4
\end{aligned}
$$


Similar calculation for other boundaries show that across a vertical boundary, going from a patch of higher density to the one of lower density, we have $\Delta m_{\mathbf{P}}=-1$ and $\Delta n_{\mathrm{P}}=0$. Across a boundary with slope $\pm 1, \Delta m_{\mathrm{P}}=0$, and $\Delta n_{\mathrm{P}}= \pm 1$.

In the outermost patch, clearly $\phi(\xi, \eta)=0$, and for this patch both $m$ and $n$ are zero. It follows that all $m_{\mathbf{P}}$ and $n_{\mathrm{P}}$ take integer values. In the following, we denote a patch by integers $(m, n)$, and write the corresponding coefficients $d_{\mathbf{P}}, e_{\mathbf{P}}$, and $f_{\mathbf{P}}$ as $d_{m, n}, e_{m, n}$ and $f_{m, n}$. With this convention, the matching conditions in Eq. (9) can be rewritten as

$$
d_{m+1, n}=d_{m, n}, \mathrm{e}_{\mathrm{m}+1, \mathrm{n}}-\mathrm{e}_{\mathrm{m}, \mathrm{n}}=\eta_{\mathrm{m}, \mathrm{n}} / 2,(\mathrm{~m}+\mathrm{n}) \text { odd }
$$

Using similar matching conditions for the boundary of patch $(m, n)$ with slope \pm 1 , we get the conditions

$$
\begin{aligned}
& d_{m, n+1}-d_{m, n}=e_{m, n}-e_{m, n+1},(m+n) \text { odd } \\
& d_{m, n-1}-d_{m, n}=e_{m, n-1}-e_{m, n},(m+n) \text { odd }
\end{aligned}
$$

We can eliminate the variables $d_{m, n}$ and $e_{m, n}$ with $(m+$ $n$ ) even using Eq.(10) and Eq.(11). Then the equations become

$$
\begin{aligned}
e_{m+2, n}-e_{m, n} & =\eta_{m, n} / 2 \\
d_{m-2, n}-d_{m, n} & =\xi_{m, n} / 2 \\
d_{m-1, n-1}-d_{m, n} & =e_{m+1, n-1}-e_{m, n} \\
d_{m-1, n+1}-d_{m, n} & =-\left[e_{m+1, n+1}-e_{m, n}\right]
\end{aligned}
$$

It is convenient to introduce the complex variables $z=$ $\xi+i \eta, M=m+i n$ and $D=d+i e$. In these variables we can write Eq.(7) as

$$
\phi(z)=\frac{1}{8} z \bar{z}+\frac{1}{8} \operatorname{Re}\left[z^{2} \bar{M}+\bar{D} z\right]+f
$$

Under a rotation of axes by an angle $\theta, z \rightarrow z^{\prime}=z e^{i \theta}$, the requirement that $\phi$ is invariant is satisfied if we have

$$
M^{\prime}=M e^{2 i \theta} ; \quad D^{\prime}=D e^{i \theta}
$$

On the $(m, n)$ lattice, with $(m+n)$ odd, the natural basis vectors are $(1,1)$ and $(1,-1)$. Let us call these $\alpha$ and $\beta$. We define the finite difference operators $\Delta_{ \pm \alpha}$ and $\Delta_{ \pm \beta}$ by

$$
\begin{aligned}
& \Delta_{ \pm \alpha} f(z)=f(z \pm \alpha)-f(z) \\
& \Delta_{ \pm \beta} f(z)=f(z \pm \beta)-f(z)
\end{aligned}
$$

Then the equations (14-15) can be written as

$$
\begin{array}{r}
\Delta_{-\alpha} d=\Delta_{\beta} e \\
\Delta_{-\beta} d=-\Delta_{\alpha} e
\end{array}
$$

These equations are the discrete analog of the familiar Cauchy-Riemann conditions connecting the partial derivatives of real and imaginary parts of an analytic function where the role of the analytic function is played by $D=d+i e$.

From Eq.(14) and Eq.(15), it is easy to deduce that $D$ satisfies the discrete Laplace's equation

$$
\left[\Delta_{\alpha} \Delta_{-\alpha}+\Delta_{\beta} \Delta_{-\beta}\right] D=0
$$

If $m$ and $n$ are large, the corresponding patch is near the origin $(|\xi|+|\eta|$ is small), and where the leading behavior of $\phi(\xi, \eta)$ is given by $\tilde{\phi}(\xi, \eta) \sim-\frac{1}{4 \pi} \log \left(\xi^{2}+\eta^{2}\right)$. Consider a point $z_{0}$, such that at $z_{0}$

$$
\partial^{2} \tilde{\phi} / \partial \xi^{2} \approx m / 4 ; \quad \partial^{2} \tilde{\phi} / \partial \xi \partial \eta \approx n / 4
$$

Then, $z_{0}$ would be expected to lie in the patch labeled by $(m, n)$. This gives $z_{0} \approx \pm(\pi \bar{M} / 2)^{-1 / 2}$. Then, setting $\partial \tilde{\phi} / \partial z$ equal to $\bar{D} / 2$ gives us

$$
D_{m, n} \simeq \pm \frac{1}{\sqrt{2 \pi}} \sqrt{m+i n}
$$

The equation (20), subjected to the behavior at large $|m|+|n|$ given by Eq. 22 on the $4 \pi$-wedge graph (for each value of $(m, n), D_{m, n}$ has two values) has an unique solution. Clearly the solution has eight fold rotational symmetry about the origin in the $(m, n)$ space. This implies that

$$
D_{-n, m}=i^{1 / 2} D_{m, n} ; \text { for all }(\mathrm{m}, \mathrm{n}) .
$$

Given $D_{m, n}$, its real and imaginary parts determine $d_{m, n}$ and $e_{m, n}$, and using Eq. 12,13 we determine the exact positions of all the patch corners. The exact eight-fold rotational symmetry of the adjacency graph of the pattern, and the fact that $D$ satisfies Eq.(20) on the adjacency graph together imply the eight-fold rotational symmetry of all the distances in the pattern.

We have not been able to find a closed-form formula for $D_{m, n}$. But the system of coupled linear equations 20 can be determined numerically to very good precision by solving it on a finite grid $-L \leq m, n \leq L$ with the condition in Eq. 22 imposed exactly at the boundary. We determined $d_{m, n}$ and $e_{m, n}$ numerically for $L=100,200,400$, and extrapolated our results for $L \rightarrow \infty$. We find $d_{1,0}=0.5000$ and $d_{2,1}=0.6464$, in perfect agreement with the exact theoretical values $1 / 2$ and $1-1 / 2 \sqrt{2}$ respectively.

Our arguments above can be extended to other two dimensional lattices, so long as there are only two allowed values of $\Delta \rho$. While this is not clear why, this seems to happen for the Manhattan lattice (Fig $2 p$ ), for initial density $1 / 2$. Also, this happens on the F-lattice, with a periodic background pattern with initial density $5 / 8$ $\left[z_{i, j}=1\right.$ if $i+j$ even, or $(i, j)$ congruent to $(0,1)$ or $(2,3) \bmod 4]$. In some other cases, like the F-lattice, with initially all sites empty, the pattern is very similar, but there are some non periodic patches in the outermost 
ring. Since the behavior of $\phi(\xi, \eta)$ in such patches is not known, the equations for $D_{m, n}$ do not close in this case.

We thank L. Levine for very useful discussions. The special features of growth pattern studied here were noted first in numerical studies by Mr. Subhendu B. Singha. DD would like to thank J. P. Eckmann for getting him interested in this problem, and B. Nienhuis for discussions.

* ddhar@theory.tifr.res.in ww. theory.tifr.res.in/ ddhar

T tsadhu@gmail.com, www.theory.tifr.res.in/ tridib

‡ schandra@tifr.res.in ww.theory.tifr.res.in/ schandra

[1] M. C. Cross, P. C. Hohenberg, Rev of Mod Phys. 65, 851
(1993).

[2] John E. Pearson, Science, New Series, Vol. 261, No. 5118 (1993), 189.

[3] L. S. Schulman and P. E. Seidon, J. Stat. Phys. 19 293(1978).

[4] D. Dhar, Physica A 369, 29 (2006).

[5] D. Dhar, arXiv:cond-mat/9909009

[6] Anne Fey-den Boer, Frank Redig, J. Stat. Phys. 130, 579 (2008).

[7] Y. Le Borgne and D. Rossin, Discr. Math., 256, 775 (2002);M. Creutz. Comput. Phys. 5198 (1991).

[8] S. H. Liu, T. Kaplan and L. J. Gray, Phys. Rev. A 42, 3207 (1990).

[9] Lionel Levine and Yuval Peres, Indiana Univ. Math. J. 57 (2008), 431-450. arXiv:math/0503251.

[10] Fractal concepts in surface growth, L. Barabasi and H. E. Stanley, Cambridge Univ. Press, Cambridge, 1995.

[11] S. Ostojic, Physica A 318187 (2003). 